\title{
Work on the neutron standards since the completion of the standards eval- uation
}

\author{
Allan Carlson ${ }^{1,2, *}$ \\ ${ }^{1}$ National Institute of Standards and Technology, Gaithersburg, MD \\ ${ }^{2}$ Brookhaven National Laboratory, Upton NY
}

\begin{abstract}
An evaluation of the neutron standards that included the neutron cross section standards was recently completed. It is important to maintain experimental programs to increase the quality and extend the database for the neutron cross section standards in order to improve evaluations of them. In this paper work will be reported on new or continuing efforts on the cross section standards including that on the $30 \mathrm{keV}$ Maxwellian averaged cross section for the ${ }^{197} \mathrm{Au}(\mathrm{n}, \gamma)$ reaction that is now a standard and is used in neutron capture cross section measurements as a standard for reactions important for astrophysics.
\end{abstract}

\section{Introduction}

Measurements of most neutron cross sections are made relative to neutron cross section standards. These standards are the basis for the neutron reaction cross section databases. The cross sections used as standards are given in Table 1. Work has been done on all of the standards since the last evaluation [1] except the ${ }^{3} \mathrm{He}(\mathrm{n}, \mathrm{p})$ reaction. In many cases the results of the new measurements are in good agreement with the standards values. Such data are still very valuable since they confirm the standards values and can possibly improve the uncertainty information. In addition to the experimental efforts, work has been done by Neudecker, et al., on updating covariances of experiments in the neutron standards database. The initial work was done on the ${ }^{239} \mathrm{Pu}(\mathrm{n}, \mathrm{f})$ cross section [38]. The work focused on helping evaluators in identifying missing or suspiciously low uncertainties and missing correlations between uncertainties of the same and different experiments when estimating covariances for measurements entering their evaluations. Also work [3] has been done on unrecognized sources of uncertainties in experimental nuclear data. Inconsistencies in data have led to the speculation that for many (if not all) experiments of a given type there exist signicant unrecognized (unknown) experimentally related sources of uncertainty that cannot be eliminated by repeated measurements using the same analysis.

\section{Recent work on the neutron cross section standards database}

Work is being done at many laboratories on improvements to the standard cross sections. Recent measurements that have been completed as well as those underway and in the design stage will be noted in this paper. Eventual careful

*e-mail: carlson@nist.gov
Table 1. Neutron cross section standards

\begin{tabular}{cl}
\hline Reaction & Standards incident neutron energy range \\
\hline $\mathrm{H}(\mathrm{n}, \mathrm{n})$ & $1 \mathrm{keV}$ to $20 \mathrm{MeV}$ \\
${ }^{3} \mathrm{He}(\mathrm{n}, \mathrm{p})$ & $0.0253 \mathrm{eV}$ to $50 \mathrm{keV}$ \\
${ }^{6} \mathrm{Li}(\mathrm{n}, \mathrm{t})$ & $0.0253 \mathrm{eV}$ to $1 \mathrm{MeV}$ \\
${ }^{10} \mathrm{~B}(\mathrm{n}, \alpha)$ & $0.0253 \mathrm{eV}$ to $1 \mathrm{MeV}$ \\
${ }^{10} \mathrm{~B}\left(\mathrm{n}, \alpha_{1} \gamma\right)$ & $0.0253 \mathrm{eV}$ to $1 \mathrm{MeV}$ \\
$\mathrm{C}(\mathrm{n}, \mathrm{n})$ & $10 \mathrm{eV}$ to $1.8 \mathrm{MeV}$ \\
$\mathrm{Au}(\mathrm{n}, \gamma)$ & $0.0253 \mathrm{eV}, 0.2 \mathrm{to} 2.5 \mathrm{MeV}, 30 \mathrm{keV} \mathrm{MACS}$ \\
${ }^{235} \mathrm{U}(\mathrm{n}, \mathrm{f})$ & $0.0253 \mathrm{eV}, 7.8-11 \mathrm{eV}, 0.15 \mathrm{MeV}$ to $200 \mathrm{MeV}$ \\
${ }^{238} \mathrm{U}(\mathrm{n}, \mathrm{f})$ & $2 \mathrm{MeV}$ to $200 \mathrm{MeV}$ \\
\hline \hline
\end{tabular}

investigation of the completed experiments will result in data that can be used in the next standards evaluation.

\subsection{The $H(n, n)$ cross section}

Neutron scattering on hydrogen is one of the most fundamental processes in nuclear physics. Both the angular distribution and integral cross section are used as standards. This standard is considered to be one of the most important neutron cross section standards. It is often referred to as the primary standard since so many standards are measured relative to it. Measurements relative to it are considered absolute measurements. Improving the accuracy of the hydrogen scattering cross section is important both for a better understanding of the nucleon-nucleon interaction and for improving the knowledge of this quantity as a neutron cross section standard.

Measurements have been made in a continuuing program at the Ohio University Accelerator Laboratory on the hydrogen angular distribution standard. The work now emphasizes the small angles in the center of mass system (CMS) at 14.9 MeV where very little data are available. This work required detection of the scattered neutrons in order to make measurements at small CMS angles. Neutrons were obtained with the $\mathrm{T}(\mathrm{d}, \mathrm{n})$ reaction 
using the Ohio University Van De Graaff accelerator. A plastic scintillator was used for detecting recoil protons. Time-of-flight was used with neutron-gamma discrimination for the measurement of the number and energy of the scattered neutrons. The scattering angle varied from 20 degrees to 65 degrees in the laboratory system in 5 degree incremental steps corresponding to a neutron energy range from $13.16 \mathrm{MeV}$ to $2.66 \mathrm{MeV}$ for the scattered neutron. Preliminary results were presented in Kornikov [4]. These results are shown in figure 1.

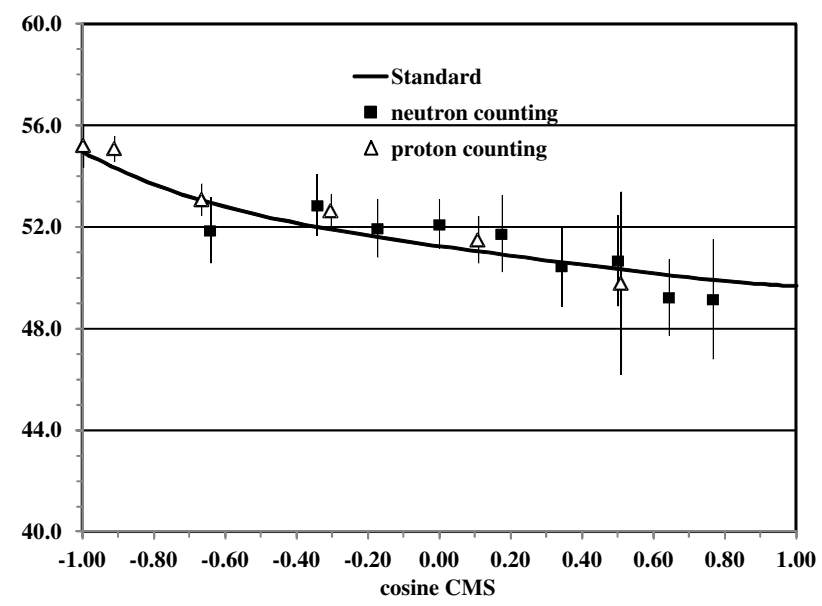

Figure 1. Comparison of the hydrogen measurements of Kornilov (shown as black boxes) with earlier Ohio work (shown as open triangles) [5] and the standards evaluation

Their earlier work [5] at $14.9 \mathrm{MeV}$ used proton recoil detection that limited the angular range to larger CMS angles. Extending the angular range by including those angles so that data can be obtained over a large angular range is important since the data are relative measurements that are normalized to the accurately known total elastic cross section. The present results and those obtained by proton detection are in excellent agreement. There is also excellent agreement with the ENDF/B-VII and ENDF/B-VIII standards evaluations within the uncertainties but there is a trend toward lower values at small CMS angles for both experiments. The results are now awaiting publishing.

Simulations are underway at the China Spallation Neutron Source [6] of the differential cross section for hydrogen scattering. The experiments will be conducted using E- $\Delta \mathrm{E}$ detector arrays in a vacuum chamber viewing a polyethlene sample bombarded by the white neutron source. Simulations have been conducted to determine what neutron energy range and positions of the detectors should be used as well as durations for the event and background measurements. It is possible that angles from 10 to 55 degrees in the laboratory system may be possible. Indications are that measurements may be possible to neutron energies considerably above $20 \mathrm{MeV}$. It is important to extend the hydrogen standard to higher neutron energies. It is now limited to $20 \mathrm{MeV}$. R-matrix analyses are underway [7] at Los Alamos National Laboratory to extend the energy range to 150 and possibly $200 \mathrm{MeV}$. It is important that the extended evaluation which includes results below
$20 \mathrm{MeV}$, has only a negligible effect on the values below $20 \mathrm{MeV}$, which are standards and can not be changed.

\subsection{The ${ }^{6} \mathrm{Li}(\mathrm{n}, \mathrm{t})$ cross section}

The ${ }^{6} \mathrm{Li}(\mathrm{n}, \mathrm{t})$ cross section is a standard that it is hoped could be extended to 2 or $3 \mathrm{MeV}$ with small uncertainties. Then measurements of neutron cross sections (or neutron fluence) when combined with the hydrogen standard could be measured from thermal to high neutron energies.

A measurement of the ${ }^{6} \mathrm{Li}(\mathrm{n}, \mathrm{t})$ cross section has been made at NIST at a sub-thermal energy [8]. It is a direct and absolute measurement of this cross section in this neutron energy range using monoenergetic neutrons. A primary effort was focused on measuring the neutron fluence accurately. It was determined with an uncertainty of $0.06 \%$ [9]. Much investigation has gone into the uncertainty of this cross section measurement. The uncertainty is mainly from the uncertainty in the ${ }^{6} \mathrm{Li}$ mass. The deposits were obtained from IRMM. The initial value obtained was in excellent agreement with the ENDF/BVIII standards evaluation. It was recently found at IRMM that the mass reported was in error. Using the new mass value produces a cross section value with an uncertainty of $0.3 \%$ that is $1 \%$ lower than the ENDF/BVIII value. Plans have been made to use Isotope Dilution Mass Spectrometry to obtain a very accurate value for the mass. The low energy (thermal) ENDF/BVIII cross section value is largely determined by three measurements made in 1970-1971. It is not clear how well they determined their ${ }^{6} \mathrm{Li}$ masses.

Measurements [10] are underway using a white neutron source, the China Spallation Neutron Source, of the differential and angle-integrated cross sections for the ${ }^{6} \mathrm{Li}(\mathrm{n}, \mathrm{t})$ reaction. The data are being obtained from $1 \mathrm{eV}$ to $3 \mathrm{MeV}$ at 80 neutron energy points. A vacuum chamber containing $15 \mathrm{E}-\Delta \mathrm{E}$ silicon solid state detector arrays (in some cases a gridded ionization chambers was used) at laboratory angles from about 20 to 160 degrees are used for the experiment. The target is an enriched (90\%) ${ }^{6} \mathrm{LiF}$ sample. The shape data are normalized to the standard cross section in the $0.1-0.4 \mathrm{MeV}$ region. The preliminary cross section results agree well with the recommended standard below $0.5 \mathrm{MeV}$. However, the results are somewhat lower than those of most evaluations in the 0.5 3.0 MeV region. The preliminary cross section results are shown in figure 2.

Work [15] was done on a ${ }^{6} \mathrm{Li}(\mathrm{n}, \mathrm{t})$ measurement at the GELINA facility at IRMM in Geel, Belgium. A twin Frischgrid ionisation chamber was employed for measuring both ${ }^{6} \mathrm{Li}(\mathrm{n}, \mathrm{t})$ and ${ }^{235} \mathrm{U}(\mathrm{n}, \mathrm{f})$ reactions in separate compartments. These measurements were made in a collaboration that included Josch Hambsch and a graduate student Kai Jansson at Uppsala University. There were a number of problems as outlined in Jansson's thesis. The final result obtained is lower than the ENDF/B cross section above $300 \mathrm{keV}$ and above $500 \mathrm{keV}$ data could not be obtained due to the $\gamma$-flash. He finally concluded more experimental work is required. The preliminary ${ }^{6} \mathrm{Li}(\mathrm{n}, \mathrm{t})$ cross section results are shown in figure 3 . 


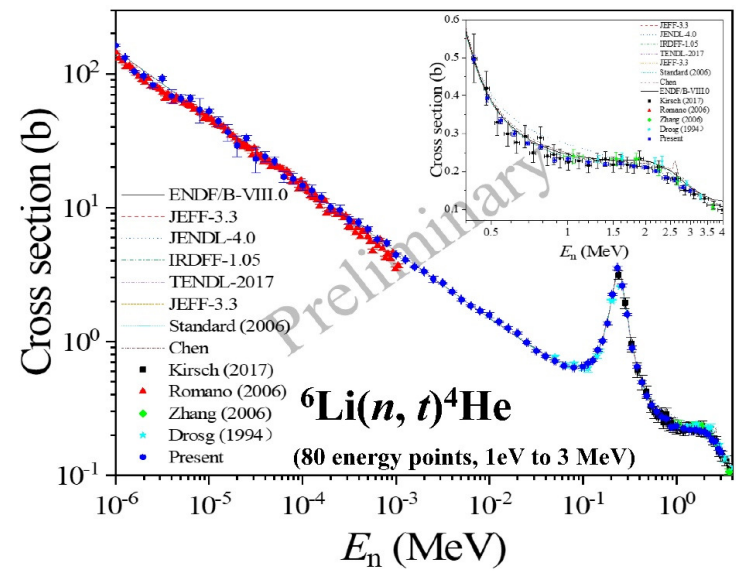

Figure 2. Measurements of the ${ }^{6} \mathrm{Li}(\mathrm{n}, \mathrm{t})$ cross section by Bai (shown as "Present") compared with several evaluations and measurements by [11], [12], [13], [14]

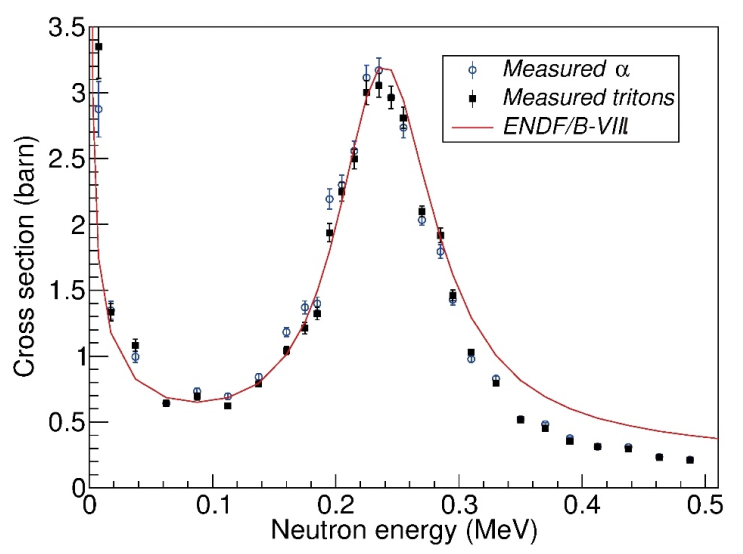

Figure 3. Measurements of the ${ }^{6} \mathrm{Li}(\mathrm{n}, \mathrm{t})$ cross section by Jansson compared with the standards evaluation

\subsection{The ${ }^{10} \mathrm{~B}+\mathrm{n}$ cross sections}

It is hoped that the ${ }^{10} \mathrm{~B}(\mathrm{n}, \alpha)$ and ${ }^{10} \mathrm{~B}\left(\mathrm{n}, \alpha_{1} \gamma\right)$ cross sections in addition to the ${ }^{6} \mathrm{Li}(\mathrm{n}, \mathrm{t})$ cross section could be improved so that their uncertainties are small in the 2 or $3 \mathrm{MeV}$ region. Then measurements of neutron cross sections could be measured from thermal to high neutron energies using those cross sections and the hydrogen standard.

Differential and angle-integrated cross sections for the ${ }^{10} \mathrm{~B}(\mathrm{n}, \alpha){ }^{7} \mathrm{Li}$ reaction have been measured by Jiang [16] at the China Spallation Neutron Source. The measurements have been made for neutron energies from $1 \mathrm{eV}$ to 2.5 $\mathrm{MeV}$ with 71 angular distributions obtained. The detector chamber used in this experiment is the same one used by Bai for the ${ }^{6} \mathrm{Li}(\mathrm{n}, \mathrm{t})$ measurements. With the $15 \mathrm{de}-$ tectors, angular distributions of a-particles were measured from about 20 degrees to about 160 degrees in the laboratory system. The data were Fit with Legendre polynomials and the relative (n, $\alpha$ ) cross-sections were obtained through integration. The absolute cross sections were normalized using the standard cross-sections of the ${ }^{10} \mathrm{~B}(\mathrm{n}, \alpha){ }^{7} \mathrm{Li}$ reaction in the $0.3-0.5 \mathrm{MeV}$ neutron energy region. It was possible to separate the cross sections for the ${ }^{10} \mathrm{~B}\left(\mathrm{n}, \alpha_{0}\right)$ and ${ }^{10} \mathrm{~B}\left(\mathrm{n}, \alpha_{1}\right)$ reactions for neutron energies up to $1 \mathrm{MeV}$. Only preliminary data are available. In figure 4 one of the angular distributions is shown. The different angular distribution obtained at $2 \mathrm{MeV}$ compared with the evaluations shown would indicate possible changes in the integrated standard ${ }^{10} \mathrm{~B}(\mathrm{n}, \alpha){ }^{7} \mathrm{Li}$ cross section at that energy.

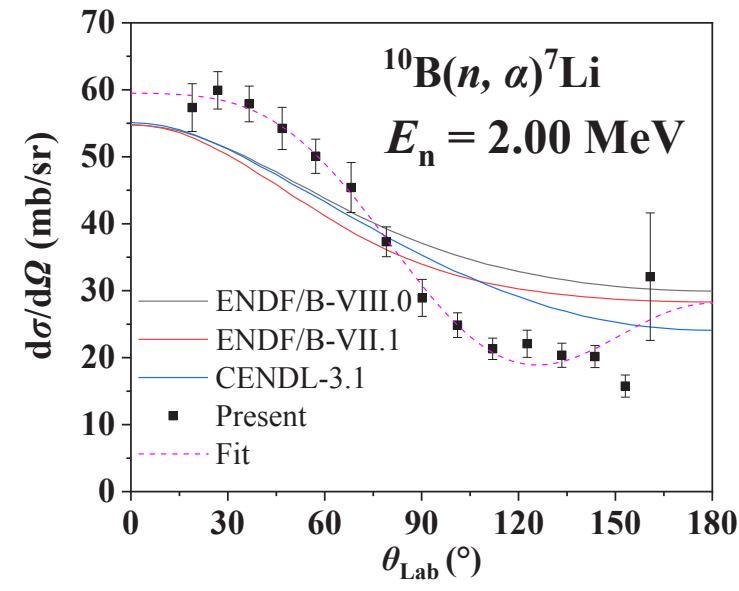

Figure 4. Measurements by Jiang of the angular distribution for the ${ }^{10} \mathrm{~B}(\mathrm{n}, \alpha)^{7} \mathrm{Li}$ reaction with $2 \mathrm{MeV}$ neutrons compared with the results from recent evaluations

Massey et al. [17] have measured ${ }^{10} \mathrm{~B}(\mathrm{n}, \mathrm{Z})$ reactions for neutron energies from 2 to $20 \mathrm{MeV}$. For the experiment thin window proportional counters and silicon detectors were used as E-DE telescopes for particle identification. Proton, triton and alpha particles were measured at four angles at the LANSCE WNR facility. Differential cross sections were obtained for the ${ }^{10} \mathrm{~B}\left(\mathrm{n}, \mathrm{p}_{0}\right){ }^{10} \mathrm{Be}$, ${ }^{10} \mathrm{~B}\left(\mathrm{n}, \mathrm{p}_{1}\right){ }^{10} \mathrm{Be},{ }^{10} \mathrm{~B}\left(\mathrm{n}, \mathrm{t}_{0}\right){ }^{8} \mathrm{Be},{ }^{10} \mathrm{~B}\left(\mathrm{n}, \mathrm{t}_{1}\right){ }^{8} \mathrm{Be},{ }^{10} \mathrm{~B}\left(\mathrm{n}, \alpha_{2}\right){ }^{7} \mathrm{Li}$, and ${ }^{10} \mathrm{~B}\left(\mathrm{n}, \alpha_{3}\right)^{7} \mathrm{Li}$ cross sections. Since all these reactions involved the same compound nucleus, they provide information on the boron standard cross sections. Through Rmatrix fits to these data, even the data beyond the energy range of the standards will have an impact on the standards. In figure 5 angular distribution measurements by Massey are shown.

Bevilacqua [18] have made measurements of the branching ratio, the angular distribution and the ${ }^{10} \mathrm{~B}(\mathrm{n}, \alpha)$ and ${ }^{10} \mathrm{~B}\left(\mathrm{n}, \alpha_{1} \gamma\right)$, cross sections relative to the ${ }^{235} \mathrm{U}(\mathrm{n}, \mathrm{f})$ standard up to $1 \mathrm{MeV}$. The data were obtained at GELINA. There appear to be some systematic problems: The branching ratio measurements look high at the highest energies. Note this ratio is independent of the neutron fluence determination. The ${ }^{10} \mathrm{~B}\left(\mathrm{n}, \alpha_{0}\right)$ and ${ }^{10} \mathrm{~B}\left(\mathrm{n}, \alpha_{1} \gamma\right)$ cross sections used to make that ratio appear to be high below about $0.5 \mathrm{MeV}$ where the cross section has been assumed to be well defined. It could be something in common such as the neutron fluence determination. They state more work is needed to complete this effort.

In figure 6 measurements made at the n_TOF facility of the ${ }^{10} \mathrm{~B}(\mathrm{n}, \alpha) /{ }^{6} \mathrm{Li}(\mathrm{n}, \mathrm{t})$ cross section ratio by Amaducci [23] for the energy region up to $170 \mathrm{keV}$ neutron energy are shown. Separate silicon detectors were used to detect the reaction products from the ${ }^{6} \mathrm{Li}(\mathrm{n}, \mathrm{t})^{4} \mathrm{He}$ and ${ }^{10} \mathrm{~B}(\mathrm{n}, \alpha){ }^{7} \mathrm{Li}$ 


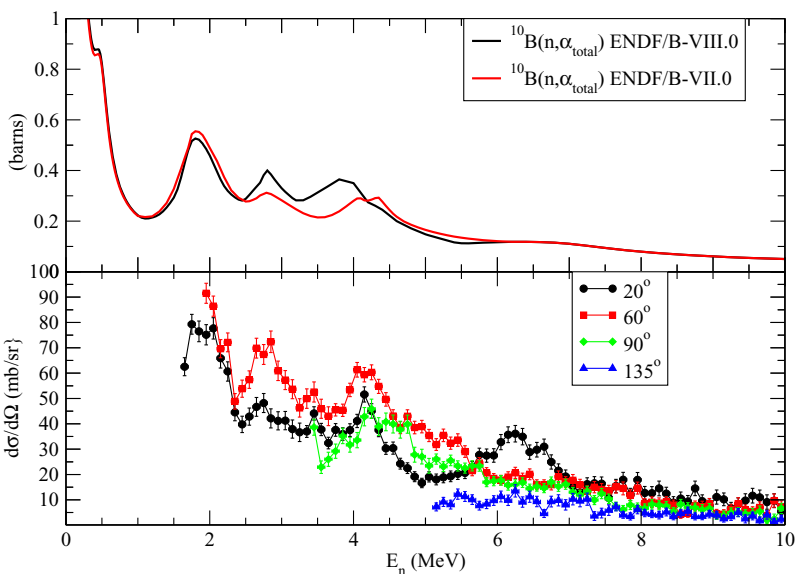

Figure 5. Angular distribution measurements of the ${ }^{10} \mathrm{~B}(\mathrm{n}, \alpha)^{7} \mathrm{Li}$ reaction by Massey in the lower part of the figure. In the upper part of the figure, the integrated cross sections for the last 2 $\mathrm{ENDF} / \mathrm{B}$ evaluations are shown for reference on the structure

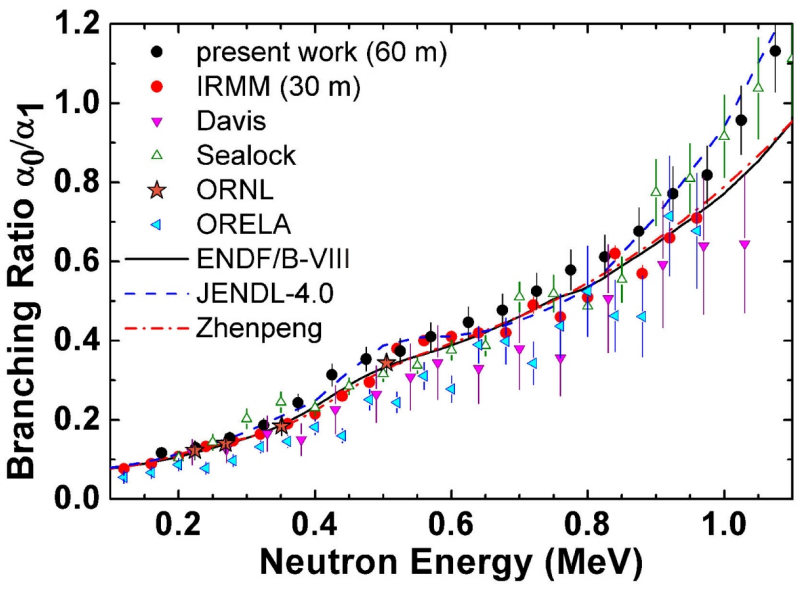

Figure 6. Measurements of the ${ }^{10} \mathrm{~B}\left(\mathrm{n}, \alpha_{0} \gamma\right) /{ }^{10} \mathrm{~B}\left(\mathrm{n}, \alpha_{1} \gamma\right)$ branching ratio by Bevilacqua compared with evaluations and experimental work by [19], [20], ORNL [21] and ORELA [22]

reactions. As can be seen in figure 7 , the data agree very well with that obtained from the standard cross sections.

\subsection{The $C(n, n)$ cross section}

The $\mathrm{C}(\mathrm{n}, \mathrm{n})$ and $\mathrm{H}(\mathrm{n}, \mathrm{n})$ are the only neutron scattering cross section standards. Measurements by Ramirez [24] have been made at the University of Kentucky of the angular distribution of neutrons scattering from carbon. Neutrons were produced with the $\mathrm{T}(\mathrm{p}, \mathrm{n})$ reaction using the $7 \mathrm{MV}$ Van De Graaff accelerator at the University of Kentucky. The data were obtained relative to the $H(n, n)$ cross section. Excellent agreement with the standards evaluation was obtained. The angle-integrated angular distributions produced total scattering cross sections that agree well with the standard values. In Fig. 8 the angular distribution results are shown compared with those from several evaluations.

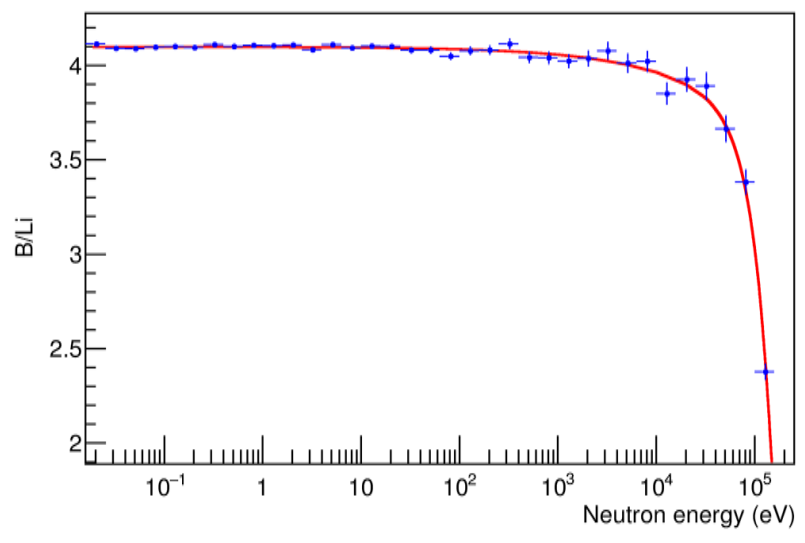

Figure 7. Measurements of the ${ }^{10} \mathrm{~B}(\mathrm{n}, \alpha) /{ }^{6} \mathrm{Li}(\mathrm{n}, \mathrm{t})$ cross section Ratio by Amaducci compared with that obtained from the standards evaluations

\subsection{The gold capture cross section}

A significant amount of work has been done on the new standard, the Maxwellian-averaged cross section (MACS) of gold capture for $\mathrm{kT}=30 \mathrm{keV}$. That cross section is a reference cross section for the KADoNiS astrophysics database [25]. The value of that reference (KADoNiS v0.3) was originally obtained from the Ratynski-Käppeler evaluation [26] based on their measurements [26] and a Macklin [27] measurement. It is $582 \pm 9 \mathrm{mb}$. This is about $5 \%$ lower than that obtained in the 2006 and 2017 standards evaluations. Several recent experiments are in very good agreement with the standards values (e.g. [28] [29], [30], [31] ).

Reifarth [32] have recently made Monte Carlo simulations that show that the effect of scattering from the copper backing in the experiment of Ratynski and Käppeler was not obtained accurately. Ratynski and Käppeler used an experimental method to deduce the value. The Monte Carlo simulations lead to a corrected experimental result consistent with the standards evaluation. In the Reifarth paper, a new evaluation is done of that MACS. They obtain $612 \pm 6 \mathrm{mb}$. This is almost identical to the new KADoNiS (v1.0) value of $611.6 \pm 6 \mathrm{mb}$ [33]. The standards value is $620 \pm 11 \mathrm{mb}$. They now agree within the standards uncertainty.

Two experiments with different methods of neutron production were recently done for the absolute determination of the $\mathrm{Au}(\mathrm{n}, \gamma) \mathrm{MACS}$ at $\mathrm{kT}=30 \mathrm{keV}$. Both experiments were done at the $3 \mathrm{MV}$ Tandem Pelletron accelerator at Seville, Spain. Activation of ${ }^{7} \mathrm{Be}$ from the ${ }^{7} \mathrm{Li}(\mathrm{n}, \mathrm{p}){ }^{7} \mathrm{Be}$ source reaction and activation of ${ }^{198} \mathrm{Au}$ from the ${ }^{197} \mathrm{Au}(\mathrm{n}, \gamma){ }^{198}$ reaction were used in the determination of the cross section for both measurements. The first experiment [34] by Jiménez-Bonilla is very similar to the Ratynski and Käppeler work but used a flat gold sample. The result obtained is $626 \pm 25 \mathrm{mb}$. The second experiment [35] is based on the production of a neutron spectrum which more closely resembles a Maxwellian than that of Ratynski and Käppeler by shaping the incident proton beam. The result obtained from this experiments is 612 


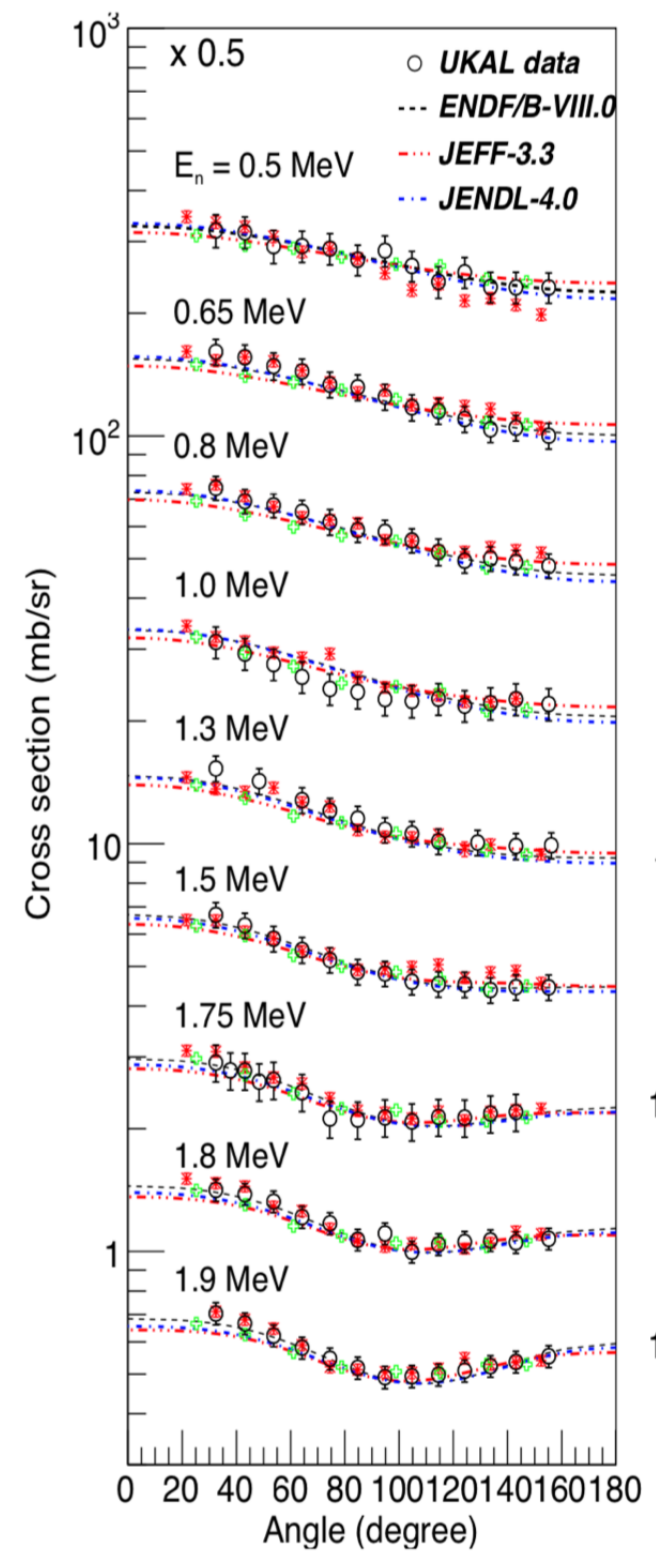

Figure 8. Angular distribution measurements (in the Center of mass system) for neutrons scattering on carbon by Ramirez. The data, labelled UKAL, are compared with several evaluations.

$\pm 20 \mathrm{mb}$. Both of these experiments used gold samples with a mass uncertainty of $1.5 \%$. Praena [36] has reduced the uncertainty to $15 \mathrm{mb}$ and $12 \mathrm{mb}$ respectively for these experiments by reducing the mass uncertainty of the samples. Thus the values are $626 \pm 15 \mathrm{mb}$ for the first method and $612 \pm 12 \mathrm{mb}$ for the second one. It should be noted that for both of these experiments an uncertainty related to spectrum correction that Ratynski and Käppeler considered as negligible is included in their values. That uncertainty is 1.4 to $1.5 \mathrm{mb}$ depending on the method used. These measurements are in excellent agreement with the standards and KADoNiS v1.0 values.

\section{$2.6{ }^{235} \mathrm{U}$ and ${ }^{238} \mathrm{U}$ fission cross sections}

New measurements of both the ${ }^{238} \mathrm{U}(\mathrm{n}, \mathrm{f}) /{ }^{235} \mathrm{U}(\mathrm{n}, \mathrm{f})$ cross section ratio and the ${ }^{239} \mathrm{Pu}(\mathrm{n}, \mathrm{f}) /{ }^{235} \mathrm{U}(\mathrm{n}, \mathrm{f})$ cross section ra- tio have been made at LANSCE by the NIFFTE collaboration. The data have been obtained using a time projection chamber. The ${ }^{238} \mathrm{U}(\mathrm{n}, \mathrm{f}) /{ }^{235} \mathrm{U}(\mathrm{n}, \mathrm{f})$ cross section ratio work has been published [37]. The cross section ratio was normalized to the ENDF/B-VIII.5 evaluation at 14.5 $\mathrm{MeV}$. This allowed the shape of the ratio to be reported over the full neutron energy range without the large neutron beam flux uncertainty introduced by the target geometry. These are the first TPC results and they are promising looking results. Overall there is good agreement with the standards evaluation. Their results are slightly lower in the medium energy region (a maximum difference of about $2.5 \%$ at about $2.4 \mathrm{MeV}$ ) but generally within the ENDF/B-VIII uncertainty . In Fig. 9, measurements of the ${ }^{238} \mathrm{U}(\mathrm{n}, \mathrm{f}) /{ }^{235} \mathrm{U}(\mathrm{n}, \mathrm{f})$ cross section ratio by Casperson are compared with recent measurements and the ENDF/BVIII. $\beta 5$ evaluation. An analysis by Neudecker indicates these results produce somewhat smaller uncertainties in the standards [38]. The ${ }^{239} \mathrm{Pu}(\mathrm{n}, \mathrm{f}) /{ }^{235} \mathrm{U}(\mathrm{n}, \mathrm{f})$ cross section ratio data is still being analyzed. Measurements are planned with thick-backed targets and back-to-back actinide deposits which will allow precise determination of the neutron beam flux and absolute normalization.

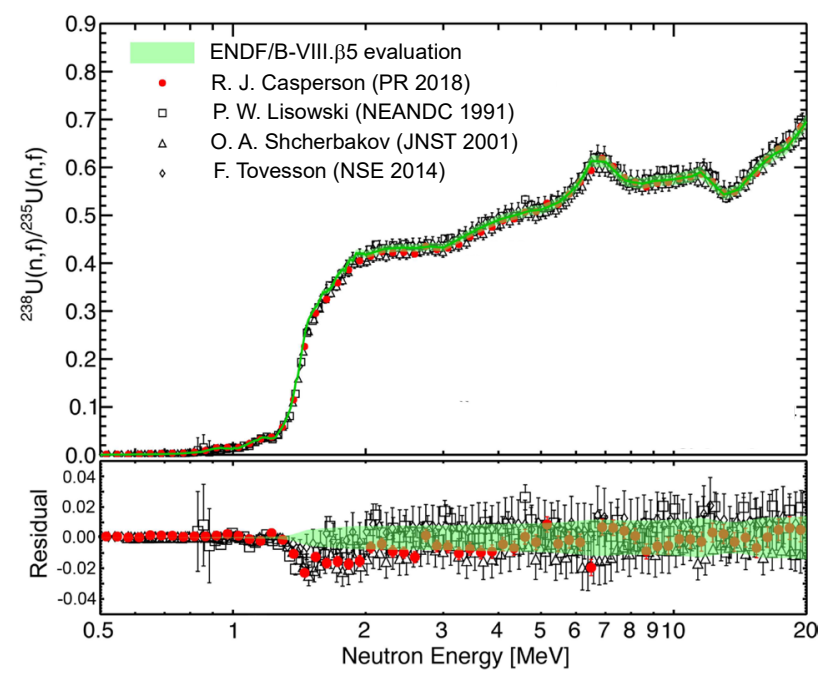

Figure 9. Measurements of the ${ }^{238} U(\mathrm{n}, \mathrm{f}) /^{235} \mathrm{U}(\mathrm{n}, \mathrm{f})$ cross section ratio by Casperson compared with those of [39], [40], [41] and the ENDF/B-VIII. $\beta 5$. Also shown re shown compared with those from several evaluations. In the lower part of the figure residuals are shown.

A measurement was made of the ${ }^{235} U(n, f)$ cross section from thermal to $170 \mathrm{keV}$ by Amaducci et al.[23] at the at the $n \_$TOF facility. The data are relative to the ${ }^{6} \mathrm{Li}(\mathrm{n}, \mathrm{t})$ and ${ }^{10} \mathrm{~B}(\mathrm{n}, \alpha)$ cross section standards. They are normalized using the 7.8 to $11 \mathrm{eV}$ fission integral. The results obtained in the standards energy region, 150-170 keV agree with the standards evaluation.

There is a need to extend a fission cross section standard to above $200 \mathrm{MeV}$. At this conference work on a measurement of the ${ }^{235} \mathrm{U}(\mathrm{n}, \mathrm{f})$ cross section from $10 \mathrm{MeV}$ to 1 $\mathrm{GeV}$ relative to the $\mathrm{n}-\mathrm{p}$ scattering cross section was presented [42]. Measurements were made at the n_TOF facil- 
ity. The experimental setup consisted of two fission detectors and three detectors to measure the neutron flux. Fission fragments were detected using both a Parallel Plate Avalanche Counter - particularly suited for neutron energies above $200 \mathrm{MeV}$ - and a Parallel Plate Ionization Chamber - designed for the energy region from $10 \mathrm{MeV}$ to $200 \mathrm{MeV}$. The number of neutrons impinging on the ${ }^{235} \mathrm{U}$ samples was measured by detecting recoil protons emitted from Polyethylene samples. Three different Proton Recoil Telescopes were used to cover the entire neutron energy region, from $10 \mathrm{MeV}$ to $1 \mathrm{GeV}$. A discussion of preliminary results was presented.

At NIST a program [43] has been started to improve the ${ }^{235} U(n, f)$ cross section at a sub-thermal energy. Well characterized ${ }^{235} \mathrm{U}$ samples combined with the excellent determination of neutron fluence [9] at the $0.06 \%$ level will provide a very accurate determination of this cross section. There are only 4 accurate measurements of this cross section in the thermal energy region. They are all made in the 1959-1965 era. It is time for additional work to be done on this important cross section.

\section{References}

[1] A.D. Carlson, V.G. Pronyaev, R. Capote, et al., Nucl. Data Sheets 148, 143 (2018)

[2] D. Neudecker, D.L. Smith, F. Tovesson, et al., Nucl. Data Sheets 163, 228 (2020)

[3] R. Capote, S. Badikov, A. Carlson, et al., Nucl. Data Sheets 163, 191 (2020)

[4] N.V. Kornilov, S.M. Grimes, T.N. Massey, et al., Nucl. Sci. Eng. 194, 335 (2020)

[5] N. Boukharouba, F.B. Bateman, A.D. Carlson, et al., Phys. Rev. C82, 014001 (2010)

[6] Z. Cui, H. Jiang, H. Bai, et al., Simulations of the Measurements of Differential Cross Sections of the $n-p$ and n-d Elastic Scattering Reactions at CSNS Back-n White Neutron Source, this conference

[7] M. Paris, Private communication (2019)

[8] A.T. Yue, Private communication (2017)

[9] A.T. Yue, et al., Metrologia 55, 460 (2018)

[10] H. Bai, H. Jiang, Z. Cui, et al., Angular Differential and Angle-integrated Cross Section Measurement for the ${ }^{6} \mathrm{Li}(\mathrm{n}, \mathrm{t})^{4} \mathrm{He}$ Reaction from $1 \mathrm{eV}$ to $3 \mathrm{MeV}$ at CSNS, this conference

[11] L.E. Kirsch, M. Devlin, S.M. Mosby, et al., Nucl. Instrum. Meth. A874, 57 (2017)

[12] C. Romano, Y. Danon, R.C. Haight, et al., NIMA 562, 771 (2006)

[13] G. Zhang, R. Cao, J. Chen, et al., Nucl. Sci. Eng. 153, 41 (2006)

[14] M. Drosg, D.M. Drake, J. Masarik, Nucl. Instrum. Meth. B94, 319 (1994)

[15] K. Jansson, Measurements of Neutron-induced Nuclear Reactions for More Precise Standard Cross Sections and Correlated Fission Properties, thesis Uppsala University (2017)
[16] H. Jiang, H. Bai, Z. Cui, et al., Measurements of Differential and Angle-integrated Cross Sections for the ${ }^{10} \mathrm{~B}(\mathrm{n}, \alpha)^{7} \mathrm{Li}$ Reaction in the Neutron Energy Range of $1 \mathrm{eV}<\mathrm{En}<2.5 \mathrm{MeV}$, this conference

[17] T.N. Massey, J. E. O’Donnell, J. Ralston, et al., Study of charged-particle emission in ${ }^{10} \mathrm{~B}(\mathrm{n}, \mathrm{x})$ reactions, to be published

[18] R. Bevilacqua, F.-J. Hambsch, M. Vidali, et al., EPJ web of conferences 146, 11047, (2017)

[19] E.A. Davis, F. Gabbard, T.W. Bonner, et al., Nuclear Physics 27, 448 (1961)

[20] R.M. Sealock, J.C. Overley, Phys. Rev. C 13, 2149 (1976)

[21] R.L. Macklin, J.H. Gibbons, Phys. Rev. 165, 1147 (1968)

[22] L.W. Weston, J.H. Todd, Nucl. Sci. Eng 109, 113 (1991)

[23] S. Amaducci, L. Cosentino, M.Barbagallo, et al., Eur. Phys. J.A. 55120 (2019)

[24] A.P.D. Ramirez, et al. private communication (2018)

[25] http://www.kadonis.org/

[26] W. Ratynski, F. Käppeler, Phys. Rev. C37, 595 (1988)

[27] R.L. Macklin, Nucl. Sci. Eng. 79, 265 (1981)

[28] A. Wallner, T. Belgya, M. Bichler, et al., Phys. Rev. Lett. 112, 192501 (2014)

[29] C. Lederer, N. Colonna, C. Domingo-Pardo, et al., Phys. Rev. C83, 034608 (2011)

[30] C. Massimi, C. Domingo-Pardo, G. Vannini, et al. Phys. Rev. C81, 044616 (2010)

[31] G. Martín-Hernández, P. Mastinu, E.M. González, et al. Phys. Rev. C99 , 034616 (2019)

[32] R. Reifarth, P. Erbacher, S. Fiebiger, et al., Eur. Phys. J. Plus 133, 424 (2018)

[33] https://exp-astro.de/kadonis 1.0/

[34] P. Jiménez-Bonilla, J. Praena, POS NIC XIII 102 (2014)

[35] P. Jiménez-Bonilla, J. Praena, J. M. Quesada, Journal of Physics: Conf. Series 940, 012044 (2018)

[36] J. Praena, private communication (2019)

[37] R.J. Casperson, D.M. Asner, J. Baker, et al., Phys. Rev. C97, 034618 (2018)

[38] D. Neudecker, Private communication (2019)

[39] P.W. Lisowski, A. Gavron, W.E. Parker, et al., Report NEANDC 305/U, 177 (1991)

[40] O.A. Shcherbakov, A. Donets, A. Evdokimov, et al., Proc. Int. Conf. on Nuclear Data For Science and Technology, Vol. 1, 7 - 12 October 2001, Tsukuba, Japan, J. Nucl. Sci. Technol., Supplement 2, 230 (2002)

[41] F. Tovesson, A. Laptev, T. S. Hill, Nucl. Sci. Eng., 178, 57 (2014)

[42] A. Manna, et al., Measurement of the ${ }^{235} U(n, f)$ Cross Section Relative to n-p Scattering up to $1 \mathrm{GeV}$, this conference

[43] M.S. Dewey, Private communication (2019) 